ARTICLE

DOI: $10.1038 / s 41467-018-07108-x$

\title{
In-situ observation of plasmon-controlled photocatalytic dehydrogenation of individual palladium nanoparticles
}

\author{
Michal Vadai ${ }^{1}$, Daniel K. Angell ${ }^{1}$, Fariah Hayee ${ }^{2}$, Katherine Sytwu ${ }^{3} \&$ Jennifer A. Dionne ${ }^{1}$
}

\begin{abstract}
Plasmonic nanoparticle catalysts offer improved light absorption and carrier transport compared to traditional photocatalysts. However, it remains unclear how plasmonic excitation affects multi-step reaction kinetics and promotes site-selectivity. Here, we visualize a plasmon-induced reaction at the sub-nanoparticle level in-situ and in real-time. Using an environmental transmission electron microscope combined with light excitation, we study the photocatalytic dehydrogenation of individual palladium nanocubes coupled to gold nanoparticles with sub-2 nanometer spatial resolution. We find that plasmons increase the rate of distinct reaction steps with unique time constants; enable reaction nucleation at specific sites closest to the electromagnetic hot spots; and appear to open a new reaction pathway that is not observed without illumination. These effects are explained by plasmon-mediated population of excited-state hybridized palladium-hydrogen orbitals. Our results help elucidate the role of plasmons in light-driven photochemical transformations, en-route to design of siteselective and product-specific photocatalysts.
\end{abstract}

\footnotetext{
${ }^{1}$ Department of Materials Science and Engineering, Stanford University, Stanford, CA 94305, USA. ${ }^{2}$ Department of Electrical Engineering, Stanford University, Stanford, CA 94305, USA. ${ }^{3}$ Department of Applied Physics, Stanford University, Stanford, CA 94305, USA. Correspondence and requests for materials should be addressed to M.V. (email: michalva@stanford.edu) or to J.A.D. (email: jdionne@stanford.edu)
} 
P hotocatalysts harvest optical energy to drive chemical reactions. They are essential for processes ranging from water purification to water splitting, air filtration, and surgical instrument sterilization. While traditional photocatalysts are comprised of semiconductors or metal complexes and clusters, pure metallic plasmonic nanoparticles have emerged as a promising new class of photocatalysts ${ }^{1-4}$. The enhanced photoreactivity of plasmonic nanoparticles is attributed to their localized surface plasmon resonances (LSPR), collective oscillations of the conduction band electrons which enable strong optical absorption and scattering in the subwavelength regime, with the ability to be tuned by the size, shape, material, and the surrounding medium of the nanoparticle.

The confined electromagnetic fields, local heat generation and efficient hot carrier excitation, that accompany plasmon resonances afford several advantages for photocatalysis. For example, the strong electromagnetic fields at the surface of plasmonic nanoparticles enhance the photon flux and can promote photopolymerization, photoisomerization and enantioselective reactions $^{5-9}$. Similarly, localized heating associated with plasmonic near-fields can increase reaction rates and potentially enable spatially dependent product formation due to nanoscale reactionrate variations ${ }^{10-13}$. Finally, hot carriers generated upon plasmon decay are known to activate bond formation and/or dissociation; these hot carriers have already been used to induce chemical reactions including hydrogen dissociation on gold nanoparticles and tautomerization of porphycene molecule, which, notably, are prohibitively challenging with conventional photocatalysts ${ }^{14-19}$.

With chemical reactions dictated by atomic and molecular interactions at the nanoscale, examining these processes with near-atomic resolution is necessary to understand photochemical processes in depth and to improve plasmonic nanoparticles for next-generation catalysts. Indeed, important and often surprising structure-function relations have been observed with single and sub-particle plasmon catalysis measurements. Utilizing techniques such as dark-field optical spectroscopy ${ }^{20}$, super-resolution microscopy ${ }^{21-23}$, and exsitu scanning electron microscopy ${ }^{24}$, researchers have monitored sitespecific reaction rates and identified the reactive sites of an individual particle, indicating large particle-to-particle variability. However, to date, most experiments do not have a spatial resolution better than $15 \mathrm{~nm}$. Additionally, many techniques require fluorescent tags, fluorescent products, or nanoparticle markers to infer the chemically active sites, thereby introducing external species whose presence could complicate the interpretation of the results. An improved experimental scheme would offer both nanometer-scale spatial resolution and direct real-time monitoring of reaction rates.

Here, we examine plasmon-driven intercalation reactions insitu and in real time with sub-2nm spatial resolution. To do so, we develop an environmental electron microscopy method that allows for simultaneous optical excitation. This capability enables all the benefits of an optical microscope together with the unrivaled spatial resolution of an electron microscope. A combination of aberration-corrected imaging, diffraction, and electron energy loss spectroscopy allow us to visualize the photoreactivity of nanoparticles while correlating chemical activity with particle structure. As a model system, we explore the dehydrogenation of individual Pd nanocubes adjacent to Au nanodiscs in an antennareactor configuration. This configuration has been recently shown useful for inducing plasmonic fields in the non-plasmonic though chemically active metal, thus promoting chemical reactions with light $^{22,23}$.

\section{Results}

Identifying distinct time-constants for reaction steps. Antennareactor pairs are fabricated in a two-step process. First, we use electron beam lithography to fabricate $\mathrm{Au}$ nanodiscs (the antennas), with diameters of $100-120 \mathrm{~nm}$, on an electrontransparent $\mathrm{Si}_{3} \mathrm{~N}_{4}$ membrane. Subsequently, we prepare singlecrystalline palladium nanocubes (the reactors) with edge length of 30-50 nm using an aqueous colloidal synthesis. Finally, the nanocubes are drop-casted onto the patterned $\mathrm{Si}_{3} \mathrm{~N}_{4}$ membrane to form antenna-reactor pairs. The sample is then mounted on a cryo-cathodoluminescence (CL) holder which allows us to illuminate the sample while imaging it in an environmental TEM (ETEM). The cryo-CL holder was designed by us in cooperation with Gatan, Inc. and consists of two parabolic mirrors in a compact configuration to fit the $5 \mathrm{~mm}$ pole piece gap of the microscope. The mirrors surround the sample and include a 500 $\mu \mathrm{m}$ wide aperture to allow the primary electron beam to pass while light is focused to/from the sample. Two optical fibers inserted into the holder enable both illumination and collection of light. Figure $1 \mathrm{a}-\mathrm{c}$ shows a schematic of the experimental setup and a micrograph of a single-crystalline $\mathrm{Pd}$ cube adjacent to an Au nanoantenna.

We study the photocatalytic phase transformation of Pd from the hydrogen-rich $\beta$ phase to the hydrogen-poor $\alpha$ phase, as schematically depicted in Fig. 1d. This phase transition is accompanied by a $\sim 3.5 \%$ contraction in lattice constant and a $\sim 2 \mathrm{eV}$ red shift in the bulk plasmon resonance, which can be readily observed via diffraction and electron energy loss spectroscopy (EELS) 25,26 . We set the temperature to $243 \mathrm{~K}$ and start by increasing the $\mathrm{H}_{2}$ pressure to allow the Pd nanocube to change its phase from $\alpha$ to $\beta$. After the new hydrogen-rich $\beta$ phase is confirmed, we gradually decrease the pressure to a setpoint just above the natural unloading pressure level and wait $\sim 30 \mathrm{~min}$ to allow the system to reach equilibrium (see Methods for details). Depending on the particle size, the unloading pressure of our Pd nanocubes ranges from $20-40 \mathrm{~Pa}^{27}$. We have optimized the electron-beam dose to ensure that it is minimally-perturbative such that the nanocubes remain stably in the $\beta$ phase without optical illumination.

Upon illumination, the particles begin to desorb $\mathrm{H}_{2}$, transitioning to their a phase. The lattice contraction results in an outward shift of the single-particle diffraction points which can be easily resolved. Figure $2 \mathrm{a}, \mathrm{b}$ shows the evolution of this transition upon illumination through a series of selected-area electron diffraction (SAED) time-snapshots from a single Pd nanocube. As seen, following illumination, the diffraction pattern initially remains in the $\beta$ phase (Fig. 2bi). After several seconds, each diffraction point splits into two points, indicating $\alpha-\beta$ phase coexistence (Fig. 2bii-iv). Finally, the $\beta$ phase point diminishes in intensity until the particle exists purely in the $\alpha$ phase (Fig. 2bv). These results indicate that the phase transformation reaction is characterized by two distinct time constants which represent the two elementary reaction steps involved in this process: the first step is the desorption of hydrogen atoms from the surface of the Pd cube; its characteristic time constant, the induction time $\tau_{\mathrm{i}}$, corresponds to the time required for the system to establish a stable new phase nucleus following illumination. The second step starts immediately after the formation of the new phase and involves the continuous diffusion of hydrogen atoms from the core of the palladium lattice; its characteristic time constant, $\tau_{\mathrm{r}}$, corresponds to the phase transformation reaction time (Fig. 2c and Supplementary Note 1).

The wavelength dependency of the time constants reveals a distinct difference between the behavior of the two, as can be seen in Fig. $2 \mathrm{~d}-\mathrm{k}$. While $\tau_{\mathrm{r}}$, the reaction time, is constant, the induction time, $\tau_{\mathrm{i}}$, is highly dependent on the excitation wavelength and exhibits two dips (corresponding to enhanced reaction rates) at $610 \mathrm{~nm}$ and $690 \mathrm{~nm}$. The dip at $690 \mathrm{~nm}$ is attributed to the excitation of the bright dipole plasmon mode of 
a

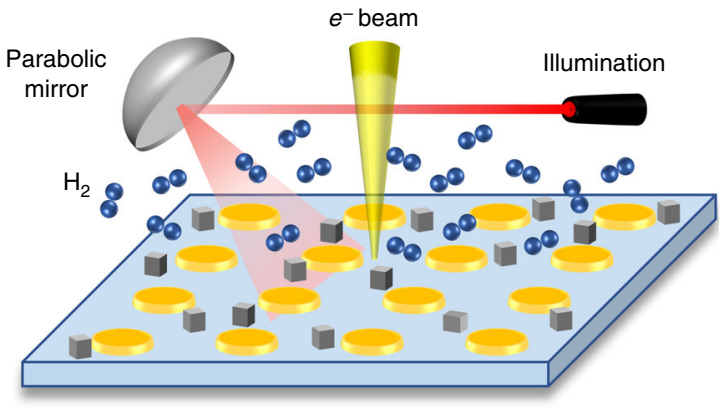

b

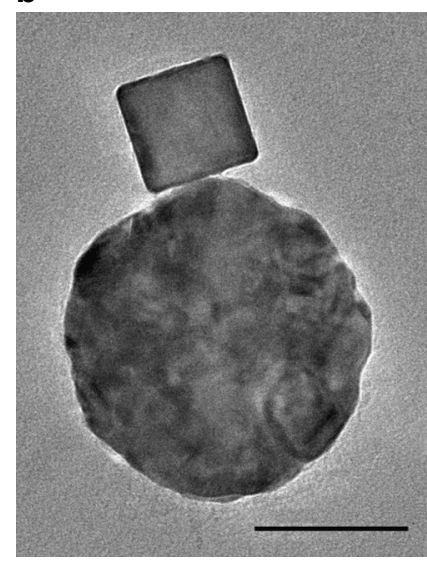

C

d

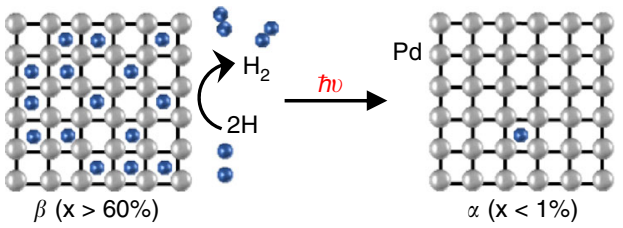

Fig. 1 Plasmon-induced phase transformation in the antenna-reactor configuration. a Schematic of the light-coupled environmental transmission electron microscope (ETEM) with an Au nanodisc array and Pd nanocubes on a $\mathrm{Si}_{3} \mathrm{~N}_{4}$ membrane. $\mathbf{b}$ Representative TEM image of an antenna-reactor pair. Scale bar is $50 \mathrm{~nm}$. c Aberration-corrected TEM image of a representative single-crystalline $\mathrm{Pd}$ nanocube on an ultrathin carbon substrate, including a zoomed-in view of the lattice and its corresponding Fourier transform. Scale bars are $10 \mathrm{~nm}$ and $2 \mathrm{~nm}$ respectively. d Schematic of the $\beta$ to $\alpha$ phase transformation reaction under study

the gold nanodisc (the antenna) ${ }^{28}$. The dip at $610 \mathrm{~nm}$ originates from a Fano-like hybridization of dark and bright plasmonic modes mediated by the substrate, enabling far-field coupling ${ }^{29,30}$. Both of these modes are apparent in our scanning-TEM-EELS measurements, as well as in our full-field simulations (Supplementary Fig. 2-4). Note that in control experiments, without the $\mathrm{Au}$ nanodiscs antennas, the $\mathrm{Pd}$ nanocubes remain loaded and show no response to the external illumination (Supplementary Fig. 5 and Supplementary Note 2). Accordingly, the wavelengthdependency we see arises purely from a plasmonic response.

The distinct wavelength dependence of the induction and reaction time constants suggests that the desorption of hydrogen from the Pd surface has a higher activation energy than the second step of the reaction, which is associated with hydrogen diffusion towards the surface and the accompanying lattice rearrangement (Supplementary Note 3). This difference in activation barriers is also found in bulk measurements with activation barriers of $\sim 5-20 \mathrm{kcal} \cdot \mathrm{mol}\left(\mathrm{H}_{2}\right)^{-1} \quad 31,32$ and $\sim 5 \mathrm{kcal} \cdot \mathrm{mol}\left(\mathrm{H}_{2}\right)^{-133}$ for the desorption and the diffusion processes respectively.
Mapping site-selectivity at plasmonic hot-spots. While SAED allows us to monitor the progression of the photocatalytic phase transformation of individual nanoparticles, it does not capture the spatial response of the system. Using STEM and TEM imaging we can map the progression of the phases in real-time with $\sim 2 \mathrm{~nm}$ spatial resolution; concurrently, we use STEM-EELS to assign regions to a particular phase. Figure $3 \mathrm{a}-\mathrm{d}$ shows how the phase transformation evolves through a series of time snapshots for two antenna-reactor pairs under illumination at $690 \mathrm{~nm}$ (Fig. 3a, b) and for two isolated Pd nanocubes under dark conditions (Fig. 3c, d) at a given pressure. As seen, the plasmon-induced phase transformation begins with the formation of an $\alpha$ phase nucleus at one edge of the particle. A (100) phase front is formed and propagates across the particle until the $\beta$ phase is completely pushed out of the particle, again through an edge of the cube. Depending on the imaging conditions, the average reaction time, $\tau_{\mathrm{r}}$, is $\sim 10 \mathrm{~s}$ for TEM imaging (measured for 21 particles in 90 unloading events) and $\sim 30 \mathrm{~s}$ for STEM imaging (measured for additional 7 particles in 18 unloading events).

Such results are remarkably distinct from the transformation that occurs in the dark. Here, we induce phase transformation by lowering the hydrogen pressure below the thermodynamic unloading pressure of the nanocubes. As seen, the reaction begins with the formation of an a phase nucleus in the corner of the particle (i.e the lowest- coordination number site), showing a phase boundary which is consistent with a (111)-interface. After nucleating, the $\alpha$ phase grows while the phase front reorients until stabilizing along the $\langle 100\rangle$ direction. The $\alpha$ phase front then propagates until the $\beta$ phase is restricted to a corner of the particle. Again, a (111)-like interface is formed, in contrast to the plasmon-mediated transformation. Interestingly, this dark phase transformation reaction is more than 10 times slower compared to the light induced one, with an average reaction time of $\sim 400 \mathrm{~s}$ (Fig. 3e). Additionally, while phase nucleation from the corner of the cube is always observed in the dark (total of 16 unloading events), optical illumination appears to enable edge nucleation as observed in $20 \%$ of the STEM measurements (total of 15 unloading events) (Fig. 3f) and in 50\% of the TEM measurements (total of 20 additional unloading events); Measurements of antenna-rector pairs in the dark also showed corner nucleation, confirming that edge nucleation is not facilitated by the presence of the antenna itself (Supplementary Fig. 6). An increased reaction rate that is beyond our temporal detection resolution could explain these observations, but it is also possible that plasmons are modifying the reaction mechanism. In particular, plasmons could lower the activation barrier for hydrogen diffusion through different crystallographic orientations, making the lowest coordination number sites not necessarily the most chemically reactive.

In addition to modifying reaction rates and mechanisms, plasmons can also enable site-selectivity through their localized strong electromagnetic fields, known as hot spots. Such siteselectivity has been observed in other recent single particle catalysis studies utilizing super-resolution microscopy ${ }^{21,23}$ and scanning electron microscopy ${ }^{24}$. To investigate site-selectivity in $\mathrm{Pd}$ dehydrogenation we correlated real time images of the unloading reaction with simulated local electromagnetic field enhancements in antenna-reactor pairs of varying separation and orientation. As seen in Fig. 4a, the electromagnetic field is highly concentrated at the surface of the Au nanodisc with a hot spot in the gap between the $\mathrm{Au}$ nanodisc and the $\mathrm{Pd}$ nanocube. We analyzed 35 phase transformation reactions in an antenna-reactor configuration and for each we labeled the cube's corners to reflect the relative distance from the hot spot (corner 1 is the closest and corner 4 is the farthest). As seen in Fig. $4 \mathrm{~b}$, upon illumination, over $31 \%$ of the particles nucleate the a phase at a corner adjacent 

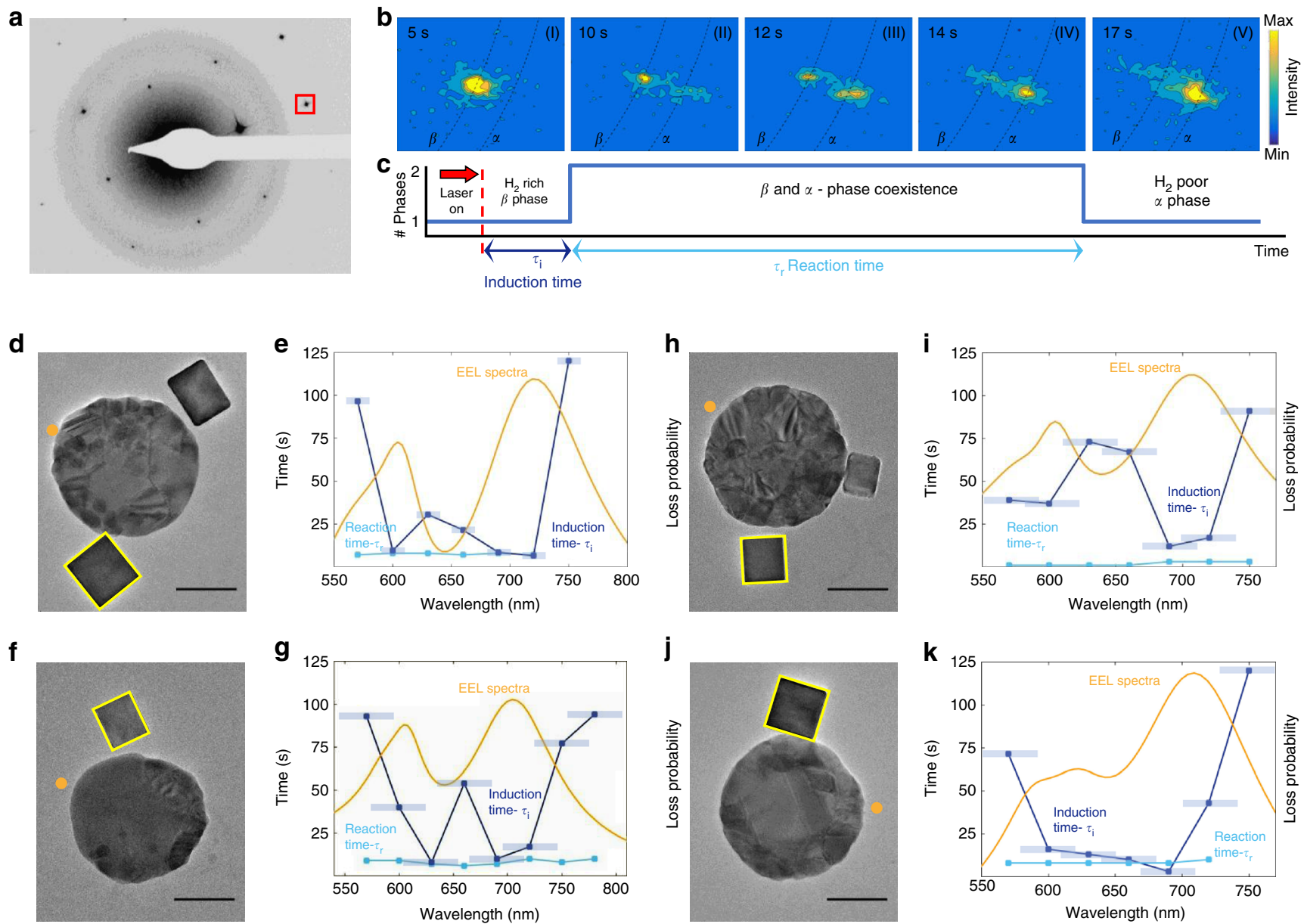

Fig. 2 Wavelength dependence of the two reaction steps. a An electron diffraction micrograph of a single Pd nanocube shown in $\mathbf{f}$. The highlighted 400 diffraction spot is monitored for the analysis of the evolution of the phase transformation reaction shown in $\mathbf{b}$. $\mathbf{b}$ Time snapshots of a zoomed-in diffraction spot showing the emerging $\alpha$ phase and the vanishing $\beta$ phase upon illumination. The black dotted lines correspond to the arc delineating constant lattice parameter (see Supplementary Fig. 1 as well). c A timeline describing the evolution of the phase transformation reaction with the two characteristic time constants, the induction time and the reaction time. $\mathbf{d}, \mathbf{f}, \mathbf{h}, \mathbf{j}$ Transmission electron microscope images of four antenna-reactor pairs with highlighted Pd cubes for which the wavelength dependence analyses appear in $\mathbf{e}, \mathbf{g}$, i and $\mathbf{k}$. Scale bars are $50 \mathrm{~nm}$. e, $\mathbf{g}, \mathbf{i}, \mathbf{k}$ Wavelength dependence of the induction time constant (dark blue) and the reaction time constant (light blue). The error bars correspond the bandwidth of the laser. Simulated EEL spectra are shown in orange with an electron beam impact position marked with a dot in the corresponding TEM images

to the hot spot while only $11.4 \%$ of the particles nucleate at the farthest corner. Without illumination, nucleation is random, and all corners desorb with equal probability $(\sim 25 \%)$.

\section{Discussion}

Plasmons can mediate chemical transformations either by enhanced light absorption, excitation of hot carriers, or by local induced heating resulting from nonradiative plasmon decay. In the antenna-reactor configuration, these phenomena can manifest themselves in either the antenna or in the reactor itself due to near-field coupling which induces a forced plasmon in the nonplasmonic material ${ }^{34,35}$. As seen in Supplementary Fig. 7, coupling in the antenna-reactor system leads to a visible-frequency absorption cross section of the Pd nanocube that rivals that of the $\mathrm{Au}$ nanodisc. First, using these absorption cross-sections, we can model the temperature of the antenna-reactor system under our experimental illumination conditions. Time-dependent simulations show a time-average increase of $\sim 1 \mathrm{~K}$ for the $\mathrm{Au}$ nanodisc and $\sim 7 \mathrm{~K}$ for the Pd nanocube. While instantaneous temperatures peak at $340 \mathrm{~K}$ and $640 \mathrm{~K}$ for the Au nanodisc and Pd nanocube, respectively, such temperatures decay back to steady-state values within $200-400$ ps. Due to the Pd particle's small size and fast thermal diffusion (thermal diffusivity of $\sim 10^{-5} \mathrm{~m}^{2} / \mathrm{s}$ ), a temperature gradient across the $\mathrm{Pd}$ nanoparticle is unlikely to form (Supplementary Note 4). Thus, while heating contributes to the enhanced kinetics of the transformation, temperature alone cannot account for the observed site-selectivity.

Photogenerated carriers can also contribute to the reaction, either through direct excitation of the molecular orbitals or through indirect excitation via hot carriers. In the latter case, hot carriers from the metal populate the antibonding adsorbate orbitals, leading to a significant displacement from the equilibrium of the adsorbate geometry as well as to a different energetic profile. As recently shown for photodesorption ${ }^{36,37}$ and photoadsorption ${ }^{15}$, the return of the system to its ground electronic state is accompanied by significant energy redistribution into translational, vibrational and rotational degrees of freedom, and consequently, destruction of the chemical bond. In bulk palladium hydrides, hybridization between the atomic orbitals of $\mathrm{Pd}$ and $\mathrm{H}$ leads to the formation of a bonding orbital with energy of $-6.5 \mathrm{eV}$ with respect to the Pd fermi level and another orbital 

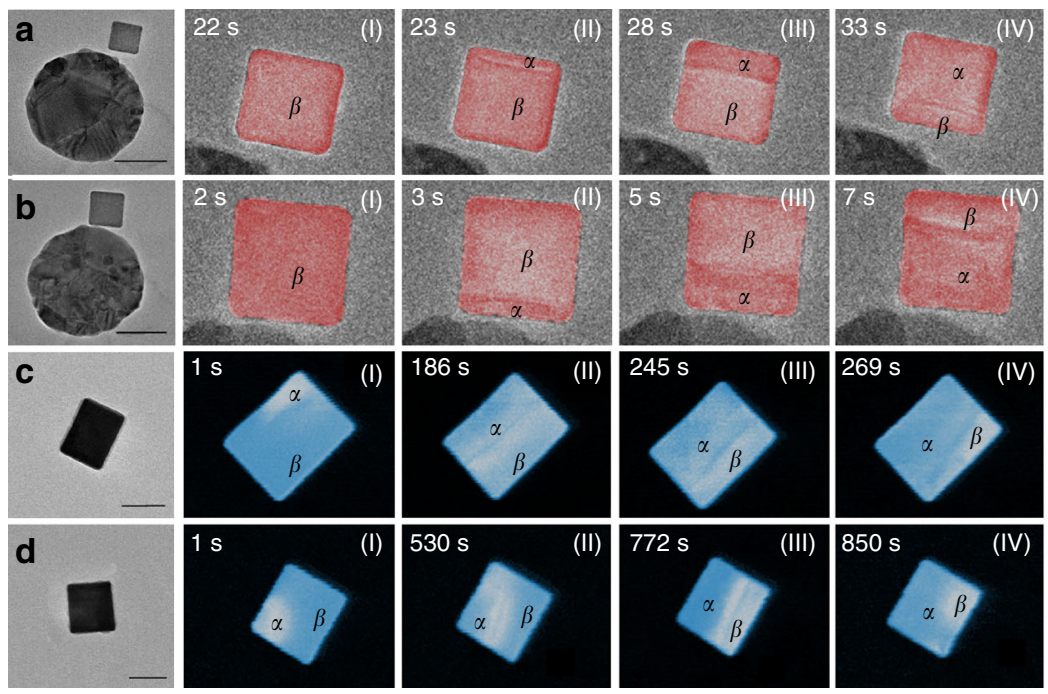
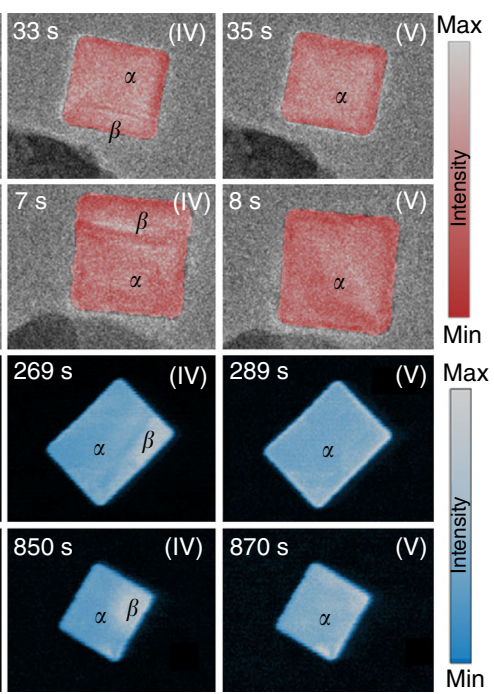

e
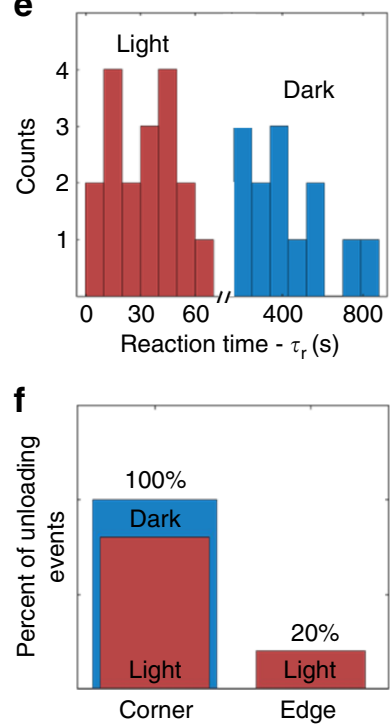

Fig. 3 Comparison between dark and light-induced phase transformation reaction. a-d Snapshots of (scanning) transmission electron microscope movies of the phase transformation reaction in the antenna-reactor (isolated) configuration with(out) illumination at $690 \mathrm{~nm}$. Scale bars are $50 \mathrm{~nm}$. e Histograms showing the distribution of reaction times for the plasmon-induced phase transformation (red) and the spontaneous phase transformation (blue) as measured in STEM mode. $\mathbf{f}$ Corner vs. edge unloading mechanism with and without illumination, also measured in STEM mode

$\mathbf{a}$

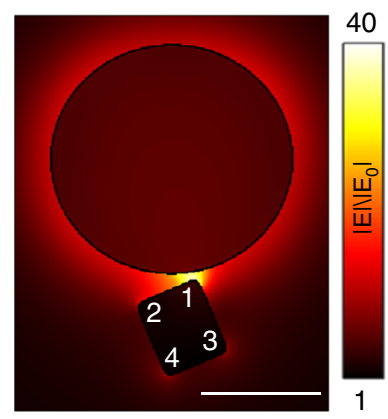

b

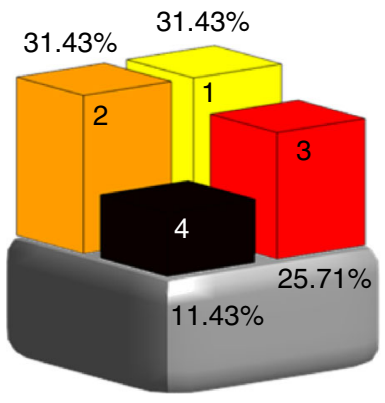

C

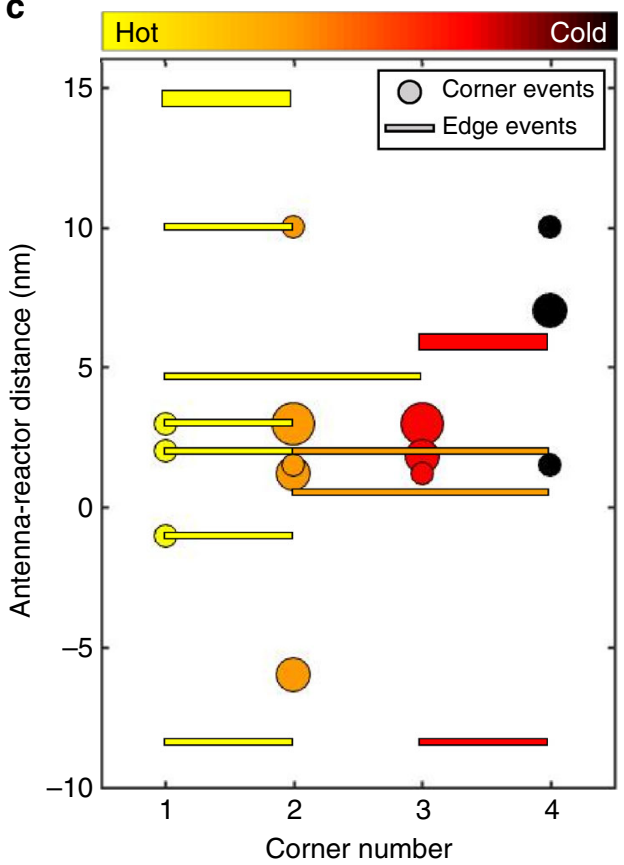

Fig. 4 The effect of electromagnetic hot spots on the phase transformation reaction. a Calculated field enhancement for the antenna (Au) - reactor (Pd) system under planewave excitation at $690 \mathrm{~nm}$. The corners of the Pd nanocube are labeled 1-4 with 1 being the corner closest to the hot spot and 4 the farthest. Scale bar is $50 \mathrm{~nm}$. b Distribution of plasmon-induced phase transformation events by corner number. c Distribution of plasmon-induced phase transformation events with respect to the distance between the nanodisc (antenna) and the nanocube (reactor). Negative antenna-reactor distance values correspond to a physical overlap between the nanodisc and the nanocube. The size of the markers corresponds to a single unloading event (small markers), 2 unloading events (medium markers) and 3 unloading events (big markers). Edge events are marked with bars spanning the two corners comprising the edge. For the summary analysis of $\mathbf{b}$, we assign each edge unloading event to one corner number (the lower corner number)

with an antibonding characteristic emerging at $2.5 \mathrm{eV}$ above the fermi level ${ }^{38-40}$. These energies could shift with nanostructuring, but given the illumination energies used in our experiment $(1.6-2.1 \mathrm{eV})$, we hypothesize that indirect charge transfer and the formation of a transient ion are not the dominant contributions to the phase transformation reaction.

On the other hand, direct absorption within the hybridized $\mathrm{Pd}-\mathrm{H}$ molecular orbitals, which does not involve charge transfer 
from the metal, is more likely to contribute to the enhanced reaction rate and site-selectivity. In this case, population of the hybridized $\mathrm{Pd}$ and $\mathrm{H}$ atom orbitals brings the system to an excited state and results in smaller activation barriers along the reaction pathway compared to the ground state (Supplementary Fig. 8$)^{41}$. Therefore, the increased absorption cross-section throughout the Pd cube expedites the phase transformation. Additionally, regions of highest absorption on the surface of the Pd nanocube, such as the hotspot, are more likely to get excited, thus promoting site-selectivity.

Since this mechanism depends on local absorption in the Pd nanocube, it is important to examine site-selectivity with respect to the antenna-reactor distance which will govern its efficiency. Figure $4 \mathrm{c}$ presents such an analysis: at overlapping antennareactor separations, where there is a physical contact between the $\mathrm{Pd}$ nanocube and the Au antenna, none of the unloading events nucleate at the cold corner, number 4 . At longer antenna-reactor distances, near field coupling with the Au antenna is less effective, leading to a smaller absorption cross section with less variation between different sites on the nanocube. In these cases, all four corners of the cube will act as local hot spots, with corner number 1 remaining the hottest due to its proximity to the antenna. In contrast to the overlapping regime, where none of the studied phase transformations began at corner number 4 , at larger antenna-reactor separations $\sim 14 \%$ of the cases show unloading from this cold corner. These results emphasize the capability of plasmons to control chemical reactions through the formation of local hot spots and highlight the future potential of enabling sitespecific reactions at the nanoscale.

In summary, we have presented an experimental method which allows us to observe and control photocatalytic reactions with sub-2nm spatial resolution spatial resolution. Using a lightcoupled environmental TEM, we are able to follow in situ and in real time the plasmon-induced dehydrogenation of individual palladium nanocubes. The $\beta$ to $\alpha$ phase transformation reaction is comprised of two elementary steps with different activation barriers, such that the excitation of plasmons affects the rate of each step differently. Under illumination, the reaction rate is enhanced by a factor of 10 compared to ambient conditions. Moreover, we find that plasmons appear to modify the reaction mechanism, within our time-resolution, allowing the $\alpha$ phase to nucleate at an edge of the cube instead of at the corners in a statistically significant number of particles. Our single particle measurements also allow us to correlate the active sites of the Pd nanocube with the formation of plasmonic hot spots, suggesting that dehydrogenation is expedited due to locally-enhanced photoabsorption and direct excitation of $\mathrm{Pd}-\mathrm{H}$ molecular orbitals. Looking forward, optical excitation and detection with an environmental TEM holds promise to explore the influence of particle structure on photocatalytic behavior for a range of reactions beyond phase transformations. For example, Raman spectroscopy and high-resolution electron-energy loss spectroscopy can be used to follow a variety of industrially-relevant reactions in the environmental TEM. As the community continues to gain better control over the variety of plasmon decay pathways there is significant promise for high-efficiency, sitespecific, and product-selective photocatalysis.

\section{Methods}

Gold nanodiscs fabrication. Au nanodiscs are fabricated on a $30 \mathrm{~nm}$ thick, $100 \mu \mathrm{m}$ wide single window, silicon nitride $\left(\mathrm{Si}_{3} \mathrm{~N}_{4}\right)$ membrane (SPI Supplies) using the following process: First, we spin coat polymethyl methacrylate (PMMA) $495 \mathrm{k}$ molecular weight $4 \%$ in anisole (MicroChem Corp.) onto the membrane at 4500 rpm for $40 \mathrm{~s}$. After baking at $180^{\circ} \mathrm{C}$ for $3 \mathrm{~min}$, the PMMA is exposed in a JEOL JBX-6300FS EBL system, followed by 1 min development in a 3:1 isopropyl alcohol (IPA): methyl isobutyl ketone (MIBK) solution. Then, we deposit a $1 \mathrm{~nm}$ adhesive layer of Ti and $30 \mathrm{~nm}$ of $\mathrm{Au}$ in KJ Lesker e-beam evaporator. After lift-off in acetone, an array of Au nanodiscs with diameter of 100-120 nm and pitch of $1 \mu \mathrm{m}$ is obtained (Supplementary Fig. 9).

Palladium nanocube synthesis. Single-crystalline $\{100\}$-terminated Pd nanocubes were synthesized following a previously reported method $^{25}$. The particles were stabilized with hexadecyltrimethylammonium bromide (CTAB) and were washed twice with water to remove excess ligands, before drop-casted on the $\mathrm{Si}_{3} \mathrm{~N}_{4}$ membrane.

Microscopy. The $\mathrm{Si}_{3} \mathrm{~N}_{4}$ membrane with the antenna-reactor nanoparticles is cleaned for one minute in an $\mathrm{Ar} / \mathrm{O}_{2}$ plasma at $50 \mathrm{~W}$ of $\mathrm{RF}$ power and then mounted on a TEM cathodoluminescence (CL)-cryogenic holder (Gatan, Inc.) that in addition to illuminating the sample, controls the temperature to $\pm 0.1 \mathrm{~K}$. To avoid condensation of contaminants on the sample during hydrogen gas flow, we use a liquid nitrogen cooled cold finger which minimizes beam-induced hydrocarbon contamination during data acquisition at all hydrogen pressures. All experiments are carried out using an FEI Titan 80-300 environmental (scanning) transmission electron microscope operated at $80 \mathrm{kV}$ for the STEM experiments and $300 \mathrm{kV}$ for high-resolution imaging and diffraction as described later. The microscope is equipped with a monochromator, an aberration corrector in the image forming objective lens and a Gatan 966 Quantum electron energy loss spectrometer. The $\mathrm{H}_{2}$ (99.9999\%, Praxair) pressure can be varied between 4 and $\sim 600 \mathrm{~Pa}$ using a home-built mass flow controller operated gas manifold system.

TEM phase transformation experiments under illumination. Unpolarized light emitted from a supercontinuum laser (SuperK Extreme, $78 \mathrm{MHz}, \sim 100$ ps pulse, NKT Photonics) is guided to the CL holder through an optical fiber (multimode, Thorlabs) and focused onto the sample by the holder-integrated parabolic mirror to form a spot size of $200 \mu \mathrm{m}$ in diameter. The bandwidth of the laser pulse is kept constant throughout the experiment and set between $\pm 10 \mathrm{~nm}$ to $\pm 25 \mathrm{~nm}$ corresponding to power levels of $1.5 \pm 0.3 \mathrm{~mW}$ and $3.8 \pm 0.3 \mathrm{~mW}$ respectively, which are measured before the entrance point to the TEM. This setting depends on the amount of power required to induce the $\beta$ to $\alpha$ phase transition reaction which is derived directly from the operating $\mathrm{H}_{2}$ pressure and its level above the pressure characterizing the nanocube's spontaneous phase transition. The diffraction patterns were obtained at $300 \mathrm{kV}$ using a $180 \mathrm{~nm}$ selected area aperture where electron dose rate is 5 electrons $/ \AA^{2}$ sec. At the beginning of the experiment, the sample is heated to $320 \mathrm{~K}$ in $500 \mathrm{~Pa}$ of $\mathrm{H}_{2}$ for $30 \mathrm{~min}$, to reduce any oxides formed during the plasma treatment. Then, the sample is cooled to $243 \mathrm{~K}$ to allow the uptake of hydrogen by the Pd nanocube and the system is let to equilibrate for $30 \mathrm{~min}$. The new hydrogen-rich $\beta$ phase of the Pd nanocube is then confirmed by comparing the selected area electron diffraction (SAED) pattern of the single particle to its original state and the pressure is reduced to $<4 \mathrm{~Pa}$ until the nanocube is unloaded again and returns to its $\alpha$ phase. This hydrogenation and dehydrogenation cycle is repeated twice in order to remove any residual strain buildup and stabilize the particle $^{25}$. Next, in order to identify the pressure level at which the Pd nanocube unloads spontaneously, we acquire a pressure-composition unloading isotherm with no illumination (Supplementary Fig. 10). We start by increasing the $\mathrm{H}_{2}$ pressure until the particle is fully loaded again, and continue by collecting a series of SAED patterns at different descending pressure points, until the particle is fully unloaded and in its hydrogen-poor, $\alpha$ phase. At each pressure point we wait $\sim 30$ min for the system to reach equilibrium before collecting the diffraction patterns. The separations between pairs of spots in the diffraction pattern are represented as percentage changes from the separation in the reference pattern of the fully loaded state. Finally, for the plasmon-induced reaction, the $\mathrm{H}_{2}$ pressure is increased to allow the particle to load once again, then we decrease the pressure to a point just above the spontaneous unloading level $(\sim+20 \mathrm{~Pa})$. After the system has reached equilibrium $(\sim 30 \mathrm{~min})$, and before illuminating the sample, the $\beta$ phase of the particle is verified again to ensure its initial state. The laser is then turned on and diffraction video is recorded for $2 \mathrm{~min}$ with temporal resolution of 4 frames/sec. We repeat this experiment for different wavelengths while keeping the power and the $\mathrm{H}_{2}$ pressure constant.

Live monitoring of the phase transformation using STEM. The sample was first heated to $333 \mathrm{~K}$ at hydrogen pressure of $473 \mathrm{~Pa}$ for $30 \mathrm{~min}$, then cooled to $238 \mathrm{~K}$, which was set constant as the operating temperature for the live-STEM monitoring of the phase transformation. The particles were cycled (hydrogenated and dehydrogenated) once before collecting any data. The bulk plasmon mode of Pd shifts from $8 \mathrm{eV}$ to $6 \mathrm{eV}$ upon dehydrogenation thus allowing us to use EELS to determine the phase of the nanocube. After making sure the particles are hydrogenated, we slowly reduced the pressure to $0 \mathrm{~Pa}$ and started monitoring particles. The monochromator was excited in the STEM mode with monochromator spot size of 15 and STEM beam size of 9 . The scan time per pixel was kept constant at $4 \mu \mathrm{s}$. The magnification was also kept constant for imaging all particles $(640 \mathrm{kX})$ to ensure similar beam dosage. The annular dark field (ADF) detector was used with a small camera length of $48 \mathrm{~mm}$, for which the collection angles are between 55 and 70 mrad. Under these conditions, the ADF detector collects only some portions of the diffraction disks from 400, 600, and 620 reflections for $<100\rangle$ zone axis. With dehydrogenation, strain and/or defects in the lattice, as well as the change in lattice 
parameter itself, result in imaging contrast as observed in the STEM scans. During the slow phase transformation, we momentarily paused the scanning to collect EEL spectrum from different contrast regions to confirm the phase at each point. The EEL spectra were collected for $0.05 \mathrm{~s}$. The movies were recorded using a screenrecording software (CamStudio), and then processed in MATLAB to enhance contrast.

Simulations. Simulations have been performed by using the boundary element method (BEM) implemented using Matlab toolbox, MNPBEM ${ }^{42}$. The optical constants of Au have been taken from Johnson and Christy ${ }^{43}$ and of PdH from Duine ${ }^{44}$. The $\mathrm{Si}_{3} \mathrm{~N}_{4}$ membrane was modeled with refractive index of 2.05 .

\section{Data availability}

All relevant data are available from the corresponding authors upon request.

Received: 15 June 2018 Accepted: 9 October 2018

Published online: 07 November 2018

\section{References}

1. Baffou, G. \& Quidant, R. Nanoplasmonics for chemistry. Chem. Soc. Rev. 43, 3898 (2014).

2. Wang, P., Huang, B., Dai, Y. \& Whangbo, M.-H. Plasmonic photocatalysts: harvesting visible light with noble metal nanoparticles. Phys. Chem. Chem. Phys. 14, 9813 (2012).

3. Linic, S., Aslam, U., Boerigter, C. \& Morabito, M. Photochemical transformations on plasmonic metal nanoparticles. Nat. Mater. 14, 567-576 (2015).

4. Zhang, X., Chen, Y. L., Liu, R.-S. \& Tsai, D. P. Plasmonic photocatalysis. Rep. Prog. Phys. 76, 046401 (2013).

5. Ueno, K. et al. Nanoparticle plasmon-assisted two-photon polymerization induced by incoherent excitation source. J. Am. Chem. Soc. 130, 6928-6929 (2008).

6. Hubert, C. et al. Near-field photochemical imaging of noble metal nanostructures. Nano Lett. 5, 615-619 (2005).

7. Volpe, G., Noack, M., Aćimović, S. S., Reinhardt, C. \& Quidant, R. Near-field mapping of plasmonic antennas by multiphoton absorption in poly(methyl methacrylate). Nano Lett. 12, 4864-4868 (2012).

8. Ho, C. S., Garcia-Etxarri, A., Zhao, Y. \& Dionne, J. Enhancing enantioselective absorption using dielectric nanospheres. ACS Photon. 4, 197-203 (2017).

9. Zhao, Y., Saleh, A. A. E. \& Dionne, J. A. Enantioselective optical trapping of chiral nanoparticles with plasmonic tweezers. ACS Photon. 3, 304-309 (2016).

10. Cao, L., Barsic, D. N., Guichard, A. R. \& Brongersma, M. L. Plasmon-assisted local temperature control to pattern individual semiconductor nanowires and carbon nanotubes. Nano Lett. 7, 3523-3527 (2007).

11. Neumann, O. et al. Solar vapor generation enabled by nanoparticles. ACS Nano 7, 42-49 (2013).

12. Hung, W. H., Aykol, M., Valley, D., Hou, W. \& Cronin, S. B. Plasmon resonant enhancement of carbon monoxide catalysis. Nano Lett. 10, 1314-1318 (2010).

13. Adleman, J. R., Boyd, D. A., Goodwin, D. G. \& Psaltis, D. Heterogenous catalysis mediated by plasmon heating. Nano Lett. 9, 4417-4423 (2009).

14. Christopher, P., Xin, H., Marimuthu, A. \& Linic, S. Singular characteristics and unique chemical bond activation mechanisms of photocatalytic reactions on plasmonic nanostructures. Nat. Mater. 11, 1044-1050 (2012).

15. Christopher, P., Xin, H. \& Linic, S. Visible-light-enhanced catalytic oxidation reactions on plasmonic silver nanostructures. Nat. Chem. 3, 467-472 (2011).

16. Mukherjee, S. et al. Hot electrons do the impossible: plasmon-induced dissociation of H2 on Au. Nano Lett. 13, 240-247 (2013).

17. Zhang, Y. et al. Direct photocatalytic conversion of aldehydes to esters using supported gold nanoparticles under visible light irradiation at room temperature. J. Phys. Chem. C 118, 19062-19069 (2014).

18. Böckmann, H. et al. Near-field enhanced photochemistry of single molecules in a scanning tunneling microscope junction. Nano Lett. 18, 152-157 (2018).

19. Zhang, X. et al. Product selectivity in plasmonic photocatalysis for carbon dioxide hydrogenation. Nat. Commun. 8, 14542 (2017).

20. Novo, C., Funston, A. M. \& Mulvaney, P. Direct observation of chemical reactions on single gold nanocrystals using surface plasmon spectroscopy. Nat. Nanotechnol. 3, 598-602 (2008).

21. Chen, G. et al. Bimetallic effect of single nanocatalysts visualized by superresolution catalysis imaging. ACS Cent. Sci. 3, 1189-1197 (2017).

22. Wilson, A. J. \& Willets, K. A. Visualizing site-specific redox potentials on the surface of plasmonic nanoparticle aggregates with superlocalization SERS microscopy. Nano Lett. 14, 939-945 (2014).
23. Zou, N. et al. Imaging catalytic hotspots on single plasmonic nanostructures via correlated super-resolution and electron microscopy. ACS Nano 12, 5570-5579 (2018).

24. Cortés, E. et al. Plasmonic hot electron transport drives nano-localized chemistry. Nat. Commun. 8, 14880 (2017)

25. Narayan, T. C. et al. Direct visualization of hydrogen absorption dynamics in individual palladium nanoparticles. Nat. Commun. 8, 14020 (2017).

26. Narayan, T. C., Baldi, A., Koh, A. L., Sinclair, R. \& Dionne, J. A. Reconstructing solute-induced phase transformations within individual nanocrystals. Nat. Mater. 15, 768-774 (2016).

27. Baldi, A., Narayan, T. C., Koh, A. L. \& Dionne, J. A. In situ detection of hydrogen-induced phase transitions in individual palladium nanocrystals. Nat. Mater. 13, 1143-1148 (2014).

28. Zorić, I., Zäch, M., Kasemo, B. \& Langhammer, C. Gold, platinum, and aluminum nanodisk plasmons: Material independence, subradiance, and damping mechanisms. ACS Nano 5, 2535-2546 (2011).

29. Zhang, S., Bao, K., Halas, N. J., Xu, H. \& Nordlander, P. Substrate-induced Fano resonances of a plasmonic nanocube: A route to increased-sensitivity localized surface plasmon resonance sensors revealed. Nano Lett. 11, 1657-1663 (2011)

30. Knight, M. W., Wu, Y., Lassiter, J. B., Nordlander, P. \& Halas, N. J. Substrates matter: influence of an adjacent dielectric on an individual plasmonic nanoparticle. Nano Lett. 9, 2188-2192 (2009).

31. Johansson, M. et al. Hydrogen adsorption on palladium and palladium hydride at 1 bar. Surf. Sci. 604, 718-729 (2010).

32. Langhammer, C., Larsson, E. M., Kasemo, B. \& Zorić, I. Indirect nanoplasmonic sensing: ultrasensitive experimental platform for nanomaterials science and optical nanocalorimetry. Nano Lett. 10, 3529-3538 (2010).

33. Kay, B. D., Peden, C. H. F. \& Goodman, D. W. Kinetics of hydrogen absorption by Pd (110). Phys. Rev. B 34, 817 (1986).

34. Swearer, D. F. et al. Heterometallic antenna-reactor complexes for photocatalysis. Proc. Natl Acad. Sci. USA 113, 8916-8920 (2016).

35. Li, K. et al. Balancing near-field enhancement, absorption, and scattering for effective antenna-reactor plasmonic photocatalysis. Nano Lett. 17, 3710-3717 (2017).

36. Harris, S. M., Holloway, S. \& Darling, G. R. Hot electron mediated photodesorption: a time-dependent approach applied to NO/Pt(111). J. Chem. Phys. 102, 8235-8248 (1995).

37. Olsen, T., Gavnholt, J. \& Schiøtz, J. Hot-electron-mediated desorption rates calculated from excited-state potential energy surfaces. Phys. Rev. B 79, 035403 (2009).

38. Paul, J.-F. \& Sautet, P. Density-functional periodic study of the adsorption of hydrogen on a palladium (111) surface. Phys. Rev. B 53, 8015-8027 (1996).

39. Chernov, I. P., Koroteev, Y. M., Silkin, V. M. \& Tyurin, Y. I. Evolution of the electron structure and excitation spectrum in palladium as a result of hydrogen absorption. Dokl. Phys. 53, 318-322 (2008).

40. Yang, X., Li, H., Ahuja, R., Kang, T. \& Luo, W. Formation and electronic properties of palladium hydrides and palladium-rhodium dihydride alloys under pressure. Sci. Rep. 7, 3520 (2017).

41. Spata, V. A. \& Carter, E. A. Mechanistic insights into photocatalyzed hydrogen desorption from palladium surfaces assisted by localized surface plasmon resonances. ACS Nano 12, 3512-3522 (2018).

42. Waxenegger, J., Trügler, A. \& Hohenester, U. Plasmonics simulations with the MNPBEM toolbox: consideration of substrates and layer structures. Comput. Phys. Commun. 193, 138-150 (2015).

43. Johnson, P. B. \& Christy, R. W. Optical constants of the noble metals. Phys. Rev. B 6, 4370-4379 (1972).

44. Von Rottkay, K., Rubin, M. \& Duine, P. A. Refractive index changes of Pdcoated magnesium lanthanide switchable mirrors upon hydrogen insertion. J. Appl. Phys. 85, 408-413 (1999).

\section{Acknowledgements}

Nano fabrication, TEM imaging and spectroscopy were performed at the Stanford Nano Shared Facilities (SNSF), supported by the National Science Foundation under award ECCS-1542152. We gratefully acknowledge support from Chi-Chang Kao at SLAC National Accelerator Laboratory and a postdoctoral fellowship from the TomKat Center for Sustainable Energy at Stanford University. K.S. was supported by the Gabilan Stanford Graduate Fellowship. D.K.A and K.S. acknowledge support by the National Science Foundation Graduate Research Fellowship (DGE-1656518).

\section{Author contributions}

M.V. and J.A.D. designed the experiments. M.V. and D.K.A performed all measurements under illumination and F.H. and K.S. performed the dark measurements and helped with the TEM setup. M.V. conducted the numerical simulations, analyzed the data, and wrote 
the initial draft of the manuscript. J.A.D. supervised the project and all authors discussed the results and contributed to the final manuscript preparation.

\section{Additional information}

Supplementary Information accompanies this paper at https://doi.org/10.1038/s41467018-07108-x.

Competing interests: The authors declare no competing interests.

Reprints and permission information is available online at http://npg.nature.com/ reprintsandpermissions/

Publisher's note: Springer Nature remains neutral with regard to jurisdictional claims in published maps and institutional affiliations. (c) (i) Open Access This article is licensed under a Creative Commons Attribution 4.0 International License, which permits use, sharing, adaptation, distribution and reproduction in any medium or format, as long as you give appropriate credit to the original author(s) and the source, provide a link to the Creative Commons license, and indicate if changes were made. The images or other third party material in this article are included in the article's Creative Commons license, unless indicated otherwise in a credit line to the material. If material is not included in the article's Creative Commons license and your intended use is not permitted by statutory regulation or exceeds the permitted use, you will need to obtain permission directly from the copyright holder. To view a copy of this license, visit http://creativecommons.org/ licenses/by/4.0/.

(C) The Author(s) 2018 